\title{
Characterizing a Device's Susceptibility to Broadband Signals: A Case Study
}

\author{
Jason B. Coder, John M. Ladbury \\ National Institute of Standards and Technology \\ M.S. 818.02, 325 Broadway \\ Boulder, CO 80305 \\ Email: jason.coder@nist.gov
}

\author{
David F. Hunter \\ CableLabs \\ 858 Coal Creek Circle \\ Louisville, CO 80027 \\ Email: d.hunter@cablelabs.com
}

\begin{abstract}
Electronic devices are commonly tested for their susceptibility to radiated signals which they may be exposed to during normal operation. A reverberation chamber is well suited to perform this type of testing because it can expose the device under test to a radiated signal from all polarization and incidence angles. Testing devices by exposing them to a narrow-band or $\mathrm{CW}$ signal has been well documented. However, with the increase in broadband communication signals, device manufacturers and users are becoming more interested in the device's performance when exposed to a broadband signal. In this case study, measurements of cable television/telecommunications equipment (i.e., set-top boxes, modems) are used to examine the potential for interference from 4G/LTE signals. We show that several difficulties arise when testing with broadband signals, particularly when measuring the incident electric field. We also examine how different device configurations (i.e., cabling and/or the use of splitters) can significantly change device performance.
\end{abstract}

\section{INTRODUCTION}

As new electronic products are developed and introduced into the marketplace their susceptibility to ambient RF signals is typically measured. One method to characterize their susceptibility is by utilizing a reverberation chamber [1]. A reverberation chamber, as opposed to an anechoic chamber, has the ability to expose the device under test (DUT) to a statistically uniform electric field. This effectively exposes the device to many signals impinging on the DUT from all incidence angles and with all polarizations. As a test signal is radiated inside the chamber, the DUT can be monitored for any change in performance.

In the past, devices were tested by means of a $\mathrm{CW}$ signal at the frequencies of interest. Historically, communications signals could be mimicked with a narrow-band signal and simple (e.g., amplitude) modulation [2]. With the advent of wi-fi and 4G/LTE signals, communications channels can now occupy $20 \mathrm{MHz}$ of bandwidth at a variety of frequencies. These wide channels can accommodate complex modulation schemes such as quadrature and amplitude modulation (QAM) and orthogonal frequency-division multiplexing (OFDM). As new parts of the frequency spectrum become available, these complex communications channels may exist over a wide

U.S. Government work not protected by U.S. copyright range of frequencies. Many customers are interested in testing their devices against more modern, broadband signals.

This issue was recently the interest of a consortium of multi-system operators (MSOs). MSOs typically use the band from $50 \mathrm{MHz}$ to $1 \mathrm{GHz}$ for their downstream communications (MSO to end user) and 5 to $42 \mathrm{MHz}$ for their upstream communications (user to MSO). These downstream bands are divided into $6 \mathrm{MHz}$ channels. There have been some recent reports that outline a possible interference problem in the range of $500-850 \mathrm{MHz}$ allegedly caused by $4 \mathrm{G} / \mathrm{LTE}$ communications [3]. As a result, MSOs are interested in quantifying any interference potential between wireless $4 \mathrm{G}$ systems that overlap with their conducted communications.

In this context, we explore methods for testing telecommunications equipment (e.g., set-top boxes (STBs), modems) in an attempt to quantify their susceptibility to broadband, 4G/LTE signals while simultaneously exchanging conducted communications at the same frequency. This may seem like a straight-forward test: radiate the devices with a 4G/LTE signal. However, we show that several difficulties arise when testing with broadband signals, and illustrate how different device configurations (i.e., cabling and/or the use of splitters) can induce a significant change in the DUT's apparent performance.

Though cable television equipment is shown here, many of the difficulties examined can apply to the susceptibility testing of any type of equipment with a radiated broadband signal.

\section{The Potential for Interference}

Intuitively, the potential for interference between a cable television system and a wireless 4G/LTE data system may seem low. The RF signals used in cable television systems are entirely conducted (either coaxial cable or fiber optic), and the 4G/LTE system is almost entirely wireless. Further, the two networks are designed to be independent of one another and exchange no data with each other. The networks can however, share the same frequency space. Currently, the overlap is small, but future auctions of spectrum in the $600 \mathrm{MHz}$ band will significantly increase the amount of overlap [4].

Given the way cable television networks and 4G/LTE networks operate, the simple fact that the two share the same frequency space does not guarantee interference. As an example, consider a 4G/LTE network operating at $819 \mathrm{MHz}$. 
Assume this wireless channel is being heavily used by a user close to a cable STB who is also watching television. If the channel being viewed (and tuned by the STB) does not occupy the same frequency space as the wireless 4G/LTE channel, the chance for interference is negligible. If the cable STB is tuned to a channel adjacent to or overlapping with $819 \mathrm{MHz}$, then the chance of interference is higher, but still low. Now consider if the tuned STB channel and the 4G/LTE channel occupy the same frequency space. In this case, the potential for interference is the greatest, but depending on the equipment and its configuration, not guaranteed.

In addition to the frequency overlap, the amplitude of the wireless signal must be sufficiently high and some amount of the signal must be coupled into the STB in order to create interference. The amplitude of the signal is proportional to the LTE device transmit power and inversely proportional to the distance between the LTE and cable devices. When the amount of interference reaches some level, the output of the STB (video signal to a television screen) will pixelate or macroblock, and video frames may be dropped and not displayed.

The simple example above illustrates that a risk may exist, but only under certain conditions. In reality, cable television channels are spread across the cable spectrum. Similarly, 4G/LTE channels are spread across a variety of frequencies depending on the network provider and geographic location. However, with large deployments of LTE devices and large installed base of STBs and cable modems, even a small probability of interference can result in hundreds of thousands of affected users.

The principles of this example also apply to a cable modem. However, in this case, when the interference exceeds some threshold, packets of information are dropped or decoded incorrectly. If the interference is severe enough, all of the incoming packets are dropped and the modem loses its connection to the cable network.

From this example, two questions arise. First, what is the strength of the electric field incident on the STB or modem when frames or packets are dropped? Second, how do we define a failure due to interference? Is it when packets begin to be dropped, or only when the user notices a problem. These questions are examined in detail in Section V.

\section{EXPERIMENT DESIGN AND MeAsurement SetuP}

With the goal of testing cable television devices for their susceptibility to interference from radiated 4G/LTE signals in mind, we devised the experimental setup shown in Figure 1. Like most radiated susceptibility tests, there is an RF signal generator outside the test chamber feeding a broadband antenna inside the chamber. For these tests, the signal generator was capable of simulating 4G/LTE signals emanating from a mobile device at a user-specified carrier frequency and channel width. To monitor the field level inside the reverberation chamber, a second broadband antenna is used and connected to a spectrum analyzer.

To simulate the DUTs being connected to a real cable network, they were fed with data from a server and RF modulator located outside the chamber. In the case of a STB, the video output was fed to a TV monitor. If the DUT was a modem, signal-quality parameters were queried directly from the device. For passive DUTs, a reference STB was used and the device was inserted between splitter and the STB.

Regardless of the DUT, the signal was split after entering the chamber as a means of monitoring the signal being input into the DUT. This splitter was typically placed as close to the DUT as possible. This provided an indication of how much interference was present on the input signal of the DUT.

The reverberation chamber used in this testing measures $4.2 \mathrm{~m} \times 3.6 \mathrm{~m} \times 2.9 \mathrm{~m}$. It is equipped with two stirrers, one spanning floor to ceiling and one spanning wall-to-wall. Both of these stirrers moved continuously during the testing of a device. While the stirrers were moving, a 4G/LTE signal was radiated into the chamber at a carrier frequency that matched the downstream channel frequency to the DUT. Three carrier frequencies were tested for each DUT: $627 \mathrm{MHz}, 711$ $\mathrm{MHz}$, and $819 \mathrm{MHz}$. These frequencies were chosen as a representative sample of existing and possible future overlap between the cable and wireless systems. In all cases, the LTE channel was fixed at $10 \mathrm{MHz}$ wide. The maximum number of resource blocks allowed in the LTE channel were used and populated with simulated data.

The output was monitored as the power of the radiated signal was stepped by a fixed increment until the DUT "failed." The definition of failure varied, based on the type of DUT. For STBs, failure was defined as when the video picture became pixelated for any period of time. For modems, failure was based on the number of uncorrectable packet errors, as counted by the DUT. Both of these definitions were chosen after careful consideration, but are not part of any standard, and thus could be different for other types of DUT. Section V describes the difficulty in defining the failure levels for different devices.

When the DUT was passive (e.g., cable or splitter), and a

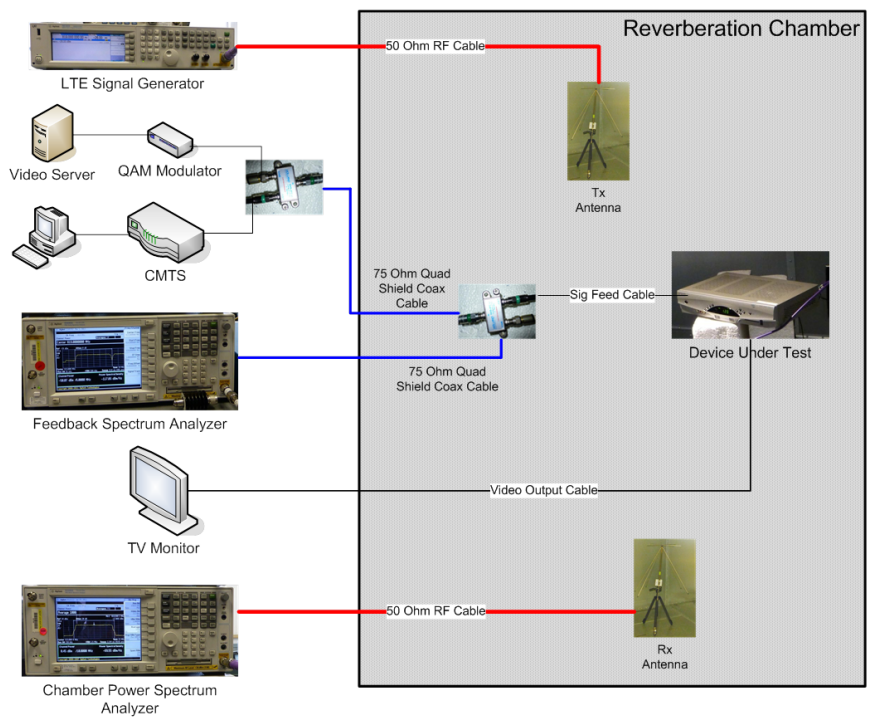

Fig. 1. Conceptual diagram of the interference test setup. 
reference STB was used, a failure was defined based on the same criteria as when the DUT was an STB.

\section{Measurement Results}

The results shown in Figures 3-6 are an estimate in terms of failure level $[\mathrm{dBmV} / \mathrm{m}]$. This level represents the electric field strength of the radiated 4G/LTE signal present inside the reverberation chamber at the time the DUT met the failure criteria. Also shown on these graphs are dotted lines representing the estimated field strength levels that a commercial cell-phone may generate, assuming an isotropic radiation pattern at a measurement distance of $1.52 \mathrm{~m}$. The upper line represents the maximum field strength corresponding to a cell phone transmitting $+23 \mathrm{dBm}$ EIRP (about $0.64 \mathrm{~V} / \mathrm{m}$ ), and the lower line represents a typical field level corresponding to a cell phone transmitting $+11 \mathrm{dBm}$ EIRP (about $0.4 \mathrm{~V} / \mathrm{m}$ ).

The field strength at the time of failure was calculated from the measured channel power from the "chamber power spectrum analyzer," shown in Figure 1. This channel power was measured over a $10 \mathrm{MHz}$ integration bandwidth that completely overlapped with the radiated 4G/LTE signal. It was also averaged as the stirrers rotated continuously. The averaged channel power measurement was then converted to an approximate field strength by use of:

$$
<E_{R}^{2}>=\frac{320 \pi^{2}}{\lambda^{2}}<P_{r e c}>[V / m],
$$

where $\lambda$ is the wavelength in meters and $\left\langle P_{\text {rec }}\right\rangle$ is the average channel power measured in watts [5].

Embedded in (1) are a few key decisions about how we conducted these measurements. Annex D of [6] requires that the maximum or average power density be used. Here, we chose to use the average field. This allows for more easily reproducible test results. This also avoids any ambiguity that might exist in [6] regarding broadband, modulated signals.

Another alternative to using the maximum or average electric field would have been to compute a power complimentary cumulative distribution function (CCDF) for the signal inside the reverberation chamber, as demonstrated in [7]. This would allow for a more realistic (in a probabilistic sense) level to be selected. Implementing this method would require a very thorough analysis as a CCDF of a time-varying signal (i.e. LTE) would need to be computed at each test frequency and each paddle position. While useful, this detailed characterization was outside the scope of this study.

Figure 2 shows a sample signal received from an antenna inside the reverberation chamber during the testing.

In addition to assessing the susceptibility of the STB and modem, we are interested in what impact (if any) the connected cable(s) or cable splitter has on the performance of the STB or modem. Both of these passive components are commonly found in the installation and use of STBs and modems. These may be part of a professional installation or added by the consumer.

\section{A. Set-top Boxes}

Seven different STB models were tested. These models ranged from legacy devices to new devices not yet deployed. In these tests, well-shielded cables were used throughout the conductive path to ensure that the results were indicative of only the DUT's susceptibility and not the shielding of the cable or splitter. Figure 3 shows the results of the STB testing. STB 1 is a legacy device that is mostly phased out and has been replaced with newer technology. STBs 2-6 represent most of the STBs currently deployed in the U.S. STB 7 is new, and has not been widely deployed.

\section{B. Modems}

Five different modem models were tested. As with the STBs, there was a mix of legacy, current, and new models. These tests were identical in setup to the STB testing. The only difference was the failure criteria, as described in Section III. Figure 4 shows the results of the modem testing. Modem 1 is a legacy device that is mostly phased out and has been replaced with newer technology. Modems 2-4 represent most of the modems currently deployed in the U.S. Modem 5 is a new modem that has not been widely deployed.

Similar to the STBs, only the legacy device fails close to the typical field strength level.

\section{Passive Splitters}

For these tests, we used a reference STB and well-shielded cables. The splitter under test was inserted between the STB and the splitter shown in Figure 1. Two retail grade and one "trade grade" splitter were tested. Figure 5 shows the results of the splitter testing.

As with the other results shown, the value shown represents the field strength level when the DUT failed. In the case of the passive devices, the failure can be attributed to the DUT instead of the STB.

Here, we see that even when using the least vulnerable STB as a reference, both retail grade splitters caused the STB to fail at a level considerably below the typical field strength level.

\section{Coaxial Cables}

Eight different types of coaxial cables were tested with a setup identical to the passive splitters. Figure 6 shows the results of the cable testing.

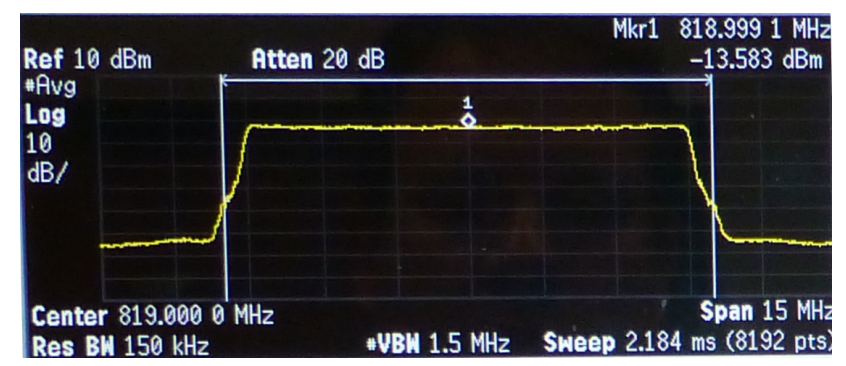

Fig. 2. A sample 4G/LTE signal received by an antenna inside the reverberation chamber. 


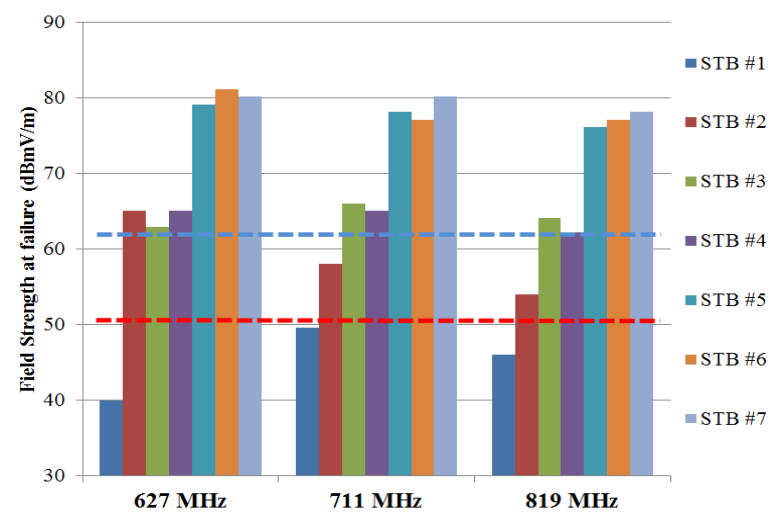

Fig. 3. Susceptibility test results for cable set-top boxes.

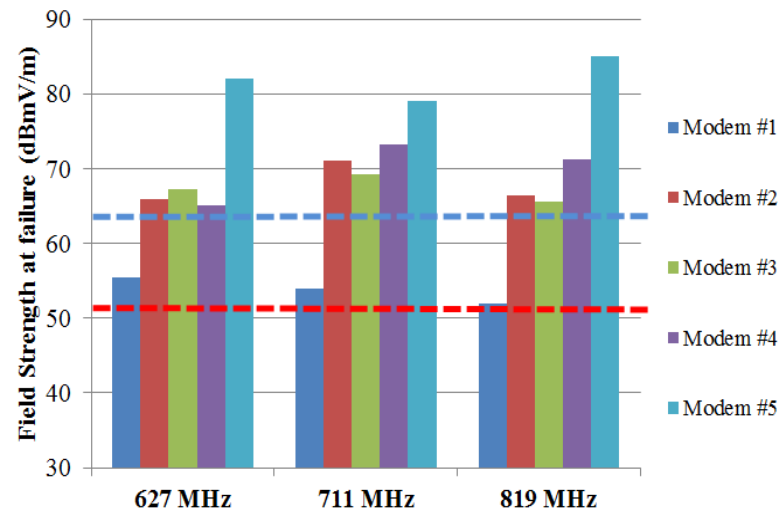

Fig. 4. Susceptibility test results for cable modems.

The coaxial cable results show some variability. The trade grade cable (layer, braided shield) performed the best. The lower performing cables were generally constructed with only a single layer of shielding. The "in-home" cable shown in red (second from the left) was a cable used by a home builder.

\section{AdDitional CONSIDERATIONS}

In light of the measurement results shown in Section IV, we should note a few key things about this measurement and its design/setup. These additional considerations are exemplified in this test, but could be easily applicable when testing other telecommunications devices.

\section{A. Definition of "Failure"}

As described in Section III, the definition of a failure was different, depending on the type of DUT. Most modern modems and STBs are equipped with some version of the data over cable service interface specification (DOCSIS) system. Devices supporting DOCSIS provide some diagnostic information about the raw signal and information coming into the device. Modern STBs and modems are capable of many different diagnostic indicators such as the number of packets received, number of packets correctly decoded, number of packet errors that were corrected, number of packet errors

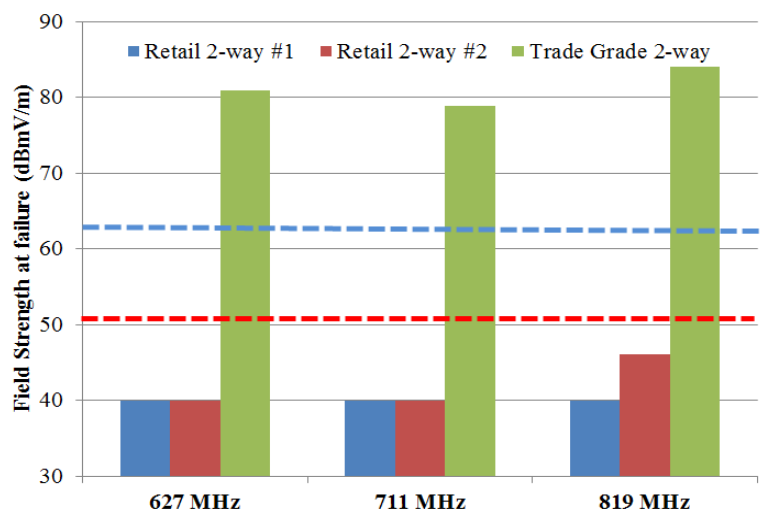

Fig. 5. Susceptibility test results for passive splitters.

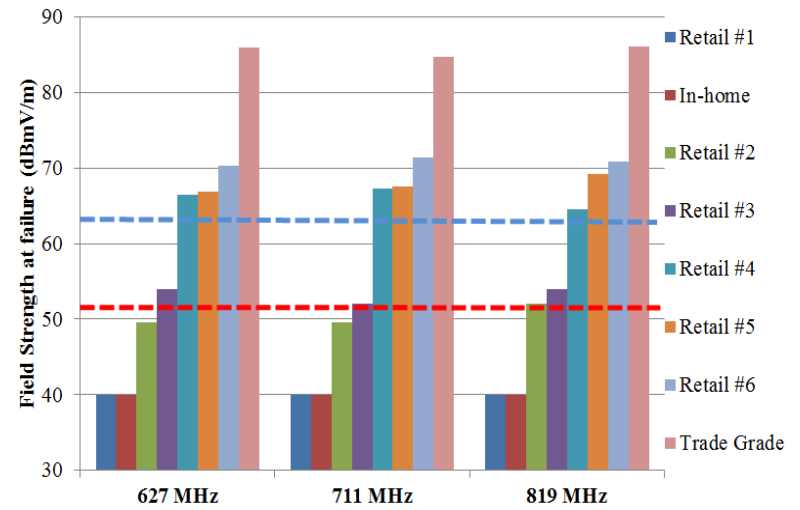

Fig. 6. Susceptibility test results for the coaxial cable testing.

with unrecoverable errors, the signal-to-noise ratio, and the coefficients used in the adaptive filter used to decode packets.

Each of these diagnostic indicators can be useful as we examine where the radiated interference is affecting the device. But, if we're interested in defining when a DUT has failed as the result of radiated interference, which of these parameters should be used? One option would be to say that none of these diagnostic measures really matter if the end-user is unable to notice a degradation in audio/video quality or data bandwidth. This definition takes full advantage of the intelligent protocols, filtering, and decoding that are built into the DUT.

A second option would be to pick one of the diagnostics, monitor it, and when it crosses a certain threshold, declare the DUT has failed due to interference. This has the advantage of being based on a single diagnostic that can be monitored as the strength of the radiated signal is increased. Whereas the first option was binary (the picture was either good or bad) monitoring a single diagnostic allows for additional information to be gathered about how the system reacts to a lower level of interference. One problem with this option is that once you pick a diagnostic, where do you set the threshold for failure? Some DUTs may have better decoding and filtering hardware/firmware and be able to tolerate more interference before the user notices an issue. Another problem with this option is that if the interference is entering the DUT from 
somewhere other than the RF input port (e.g., being received by a PCB trace or power line), it may still corrupt the output to the user, while not showing up in the diagnostic indicators.

Both the STB and modem can be tested with either of these methods. However, we choose to test the STB based on the quality of the output signal (i.e., a test pattern) as viewed by a user because it provides a way to evaluate the STB as a whole, and not just its ability to decode a signal. We choose to evaluate modems based on the number of uncorrectable errors because it provided a more rigorous metric than asking a user if they perceive a change in the data speed as the modem is radiated with an interfering signal.

Regardless of the type of DUT, the definition of a failure is an important consideration that should be carefully defined. For the reasons described above, we need to understand where in the hardware chain the failure metric is acquired.

\section{B. Traditional EMC Testing}

In traditional EMC testing, a $\mathrm{CW}$ or narrow-band signal is radiated in an anechoic environment at a fixed distance from the DUT (e.g., $3 \mathrm{~m}$ ). The strength of the radiated field is measured by a calibrated field probe in the vicinity of the DUT. The source signal is radiated from an antenna of known gain and pattern [2].

For our purposes, this test method presents a few challenges. First, the use of a free-space-like environment means that the DUT will not be exposed to the radiated electric field at all incidence angles and polarizations unless mechanically rotated. Second, calibrating an electric field probe against a 4G/LTE signal is difficult because the response of any probe circuitry to the rapid variations found in a broadband communications signal may not be known.

All of the STBs and modems measured here were tested by their respective manufacturers with a method very similar (if not identical) to what is described in IEC 61000-4-3. The radiated field strength used in testing varied depending on the device. Newer devices were tested to levels of $10 \mathrm{~V} / \mathrm{m}$ or more. All of the manufacturer testing was done with the same $1 \mathrm{kHz}$ wide, $80 \%$ modulated AM signal, specified in [2].

There have been some instances of EMC testing using bandlimited white Gaussian noise as the test signal. This may turn out to be a valid replacement for the LTE signal used here, but there was a specific interest on part of the MSOs regrading the effects of LTE.

\section{Server/Modulator Interference}

Referring back to Figure 1, we may notice that the only equipment inside the reverberation chamber (and thus shielded from the signals in the laboratory outside the chamber) are the DUT and associated cabling. This setup assumes that the server, modulator, and any other equipment used to feed the DUT are immune to the type of interference being examined in the testing. As a precaution, anyone in the lab during the testing was asked to turn their cell phone off. However, ambient signals from outside the lab were still present. We have no reason to believe the server and modulator used in this testing were particularly susceptible to $4 \mathrm{G} / \mathrm{LTE}$ interference, but we cannot say for sure.

If the server and modulator used were susceptible to 4G/LTE interference, a corrupted signal could be fed to the DUT, thus improperly biasing the results for that DUT.

\section{Viewing an Output From the DUT}

If the failure metric for a given DUT is based on a signal being output from the DUT, consideration must be given as to how the output of the DUT is fed outside the chamber. Consider the case where a STB is being tested, and the failure metric is based on the video output as viewed on a TV monitor. If the cable connecting the DUT to the TV monitor were poorly shielded, it could be susceptible to the radiated interference signal and bias the test results. This bias would be independent of the performance of the DUT.

In some testing scenarios, the cable connecting the DUT output to the monitoring device (TV monitor for a STB, computer for a modem), may be considered part of the overall DUT. Here, we attempted to isolate the susceptibility of the DUT from its input or output cables.

For the testing of cable telecommunications equipment described here, there were three different possible connections from the DUT to the TV monitor: F-type coaxial, composite, and HDMI. Due to the large technological age span of the STBs being tested, not all STBs had all three outputs. All of the STBs did have a composite video output so this was chosen for consistency. Preliminary testing indicates that the output type has no significant impact on DUT performance.

\section{E. Consequences of Using a Reverberation Chamber}

The choice to use a reverberation chamber instead of an anechoic chamber for this testing is not without consequence. The advantage of the use of a reverberation chamber is that the patterns and gains of antennas and devices used in the chamber become less important. This is also potentially a disadvantage; i.e., we would like to know where the signal is coupling into the DUT. Testing the DUT in an anechoic chamber at a variety of orientations and polarizations would be required to fully evaluate the susceptibility of the DUT.

One disadvantage of using a reverberation chamber is that it is difficult to determine the exact field strength at the time and location the DUT fails. This is partially due to the fact that stirrers were continuously rotating during the testing. Between the continuously moving stirrers and the unknown reaction time of the STBs and modems, we are unable to pinpoint the exact field strength at the time of failure. Because of this, equation (1) reflects an average field value. An alternate option would be to the maximum field and to appropriately adjust (1). This would likely cause very different results. Given that the 4G/LTE signal is a complex, modulated signal, combined with its use in a stirred chamber, the difference between the average and maximum can be large $(\sim 10 \mathrm{~dB})$.

The stirrers could have been discretely stepped, but the increased test time would have been unreasonable, given the number of devices to be tested. Testing took minutes per 
device when the stirrers were moved continuously. Stepping the stirrers would result in several changes to the testing procedure, and increase the test time from minutes to more than an hour or more per device.

There is also an issue of how the electric field inside a reverberation chamber is measured. Should the electric field be calculated as the Cartesian field or the total (RSS) field? Either definition could be used, but as results from different laboratories or test methods are compared, it's important to be clear which definition is used.

The impact the coherence bandwidth of the reverberation chamber has on the results of these measurements is unknown, and will be the subject of future research. In general, it is not considered to be a factor in traditional susceptibility measurements.

\section{F. In-band vs. Out of band Interference}

The measurement setup described here considers only the case where the radiated 4G/LTE signal completely overlaps the cable channel. The DUTs were not characterized for interference outside the tuned channel, nor any kind of interference from the wireless and cable channels being adjacent to each other. Given that a 4G/LTE signal could have three times the bandwidth of a cable channel, a single LTE channel could potentially interfere with up to three cable channels. This is an extremely unlikely scenario, but it does raise the question: "How much 4G/LTE overlap does there need to be on a cable channel?". It is assumed that if there is zero overlap between the wireless channel and the cable channel, the field level at which the DUT fails would be significantly higher than what is shown in the above graphs.

Something else to consider is whether or not the DUT (cable devices or otherwise) is digitizing more than one frequency/channel at a time. Some new cable devices have the ability to tune multiple channels simultaneously. While one channel is being viewed by the user, the other may be digitized and stored for future viewing. As this technology continues to evolve, new cable devices may be able to continuously digitize the entire cable spectrum. This ability would change the way "in-band" interference testing is viewed.

\section{DISCUSSION}

One unique aspect of testing cable devices is that their configuration can change dramatically over time. Typically, they are installed by a properly trained technician in the user's home or business. However, after the installation, the user is free to change the configuration (e.g., move the STB or modem to another room). Users may accomplish this by adding additional coaxial cable or by adding a splitter. As was shown in Section IV, if the user does not choose their cable and splitter carefully, this can have a detrimental effect on the performance of the STB or modem. The fact that the user (who is generally untrained in the principles of electromagnetic shielding) has this much control over the configuration makes mitigating any interference complicated.
The results and discussion of the test method used may hint at a larger problem looming in the distance: how can new electronic devices be tested against modern, broadband communication signals? Are the existing standards adequate for this type of testing? There is no easy answer to these questions. The method used here may by one solution, but there are still questions that need to be answered. Chiefly, how should the electric field measurement be defined for a broadband, complex, modulated signal? Such questions may need to be answered soon. As the number of wireless electronic devices continues to increase at a rapid rate, more interactions between two independent technologies (e.g., between cable telecommunication and wireless $4 \mathrm{G} / \mathrm{LTE}$ ) may be observed. Solving or characterizing these interactions will be difficult without a well defined and understood test method.

\section{CONCLUSION}

A measurement method for testing the susceptibility of some types of telecommunications devices against a radiated, broadband 4G/LTE signal in a reverberation chamber has been described. As a case study, testing has been performed on a number of commercial cable set-top boxes, modems, and passive devices. Results indicate that in general, cable telecommunication devices are adequately shielded, but this shielding can be compromised by a poor coaxial cable and/or a poorly shielded splitter. Special considerations have been outlined that highlight the difficulties when performing radiated testing using a broadband signal against any type of device.

\section{REFERENCES}

[1] M.L. Crawford and G.H. Koepke, "Design, evaluation and use of a reverberation chamber for performing electromagnetic susceptibility/vulnerability measurements," National Bureau of Standards, Tech. Note 1092, 1986.

[2] International Electrotechnical Commission Standard 61000-4-3, Edition 3.2, April, 2010.

[3] Analysis of interference to cable television due to mobile usage in the Digital Dividend, University of Twente/Radiocommunications Agency, Netherlands, 2010.

[4] T. Wheeler, "The Path to a Successful Incentive Auction," http://www.fcc.gov/blog/path-successful-incentive-auction-0, accessed December 15th, 2013.

[5] J. Ladbury, G. Koepke, and D. Camell, "Evaluation of the NASA Langley Research Center Mode-Stirred Chamber Facility," National Institute of Standards and Technology Tech. Note 1508, January 1999.

[6] International Electrotechnical Commission Standard 61000-4-21, Edition 2.0, January, 2011.

[7] P. Kaur, R. Singh, "Complementary Cumulative Distribution Function for Performance Analysis of OFDM Signals," IOSR Journal of Electronics and Communications Engineering, Vol. 2, Issue 5, Oct. 2012. 